\title{
Measuring gambling knowledge in adolescents: The construction of a new short scale for research and practice
}

\author{
Maria Anna Donati ${ }^{*}$, Carola Beccari ${ }^{2}$, Angela Biganzoli $^{3}$, Mariapaola Tadini $^{3}$, Daniela Capitanucci ${ }^{3}$, Roberta Smaniotto $^{3}$ and Caterina Primi $^{2}$ \\ ${ }^{1}$ Department of Developmental and Social Psychology, Sapienza University of Rome, Rome, Italy \\ ${ }^{2}$ Department of Neuroscience, Psychology, Drug, and Child's Health (NEUROFARBA), Section of Psychology, University of Florence, Italy \\ ${ }^{3}$ A.N.D. - Azzardo e Nuove Dipendenze", Varese, Italy.
}

\begin{abstract}
Several studies show that many adolescents gamble and a considerable proportion of them develop pathological gambling behavior. It has been shown that adolescents often have erroneous gambling knowledge, for example they are not aware of the technical definition of gambling activities or perceive gambling as a social and recreational activity. Nevertheless, nowadays there is a lack of measurement tools with adequate psychometric properties to assess gambling knowledge in adolescents. For this reason, the aim of the present study was to develop a new instrument able to evaluate this specific construct with a sample of Italian adolescents, the Gambling Related Knowledge Scale - For Adolescents (GRKS-A). In order to develop the scale and test its psychometric properties, 445 Italian adolescents participated in the study. The final version of the scale was composed of 8 items. Analyses confirmed the adequacy of the one-factor model and the reliability of this short scale. Support for the validity was also provided by obtaining significant and negative correlations with cognitive distortions, gambling economic perception, and gambling frequency. Moreover, the additional predictive power of GRKS-A on gambling frequency - with respect to the other variables - was demonstrated. Overall, findings support the suitability of the GRKS-A to measure gambling-related knowledge in research and practice involving adolescents.
\end{abstract}

\section{Introduction}

Gambling takes place when an item of value, usually money, is staked on the outcome of an event that is to some degree, unpredictable [1]. In gambling, the primary task of gamblers is to use information to try to predict the outcome of an event that is essentially unpredictable [2]. Although gambling is prohibiting for youth aged less than 18 years in most countries, several studies show that gambling is a popular activity among adolescents [3-5], who often are not aware of the detailed definition of gambling activity, perceive gambling as a recreational and social activity, and they do not feel concerned about the potential negative consequences derivable from gambling [6]. Additionally, young gamblers are prone to some errors when face to gambling, and these errors and misconceptions are often reinforced by unrealistic views of gambling given by media through advertisement that can lead young people to believe that gambling is all fun and excitement, and that it is an easy way to win big money [2]. For this reason, a proportion of youth comprised between 0.2 and 12.3 develop negative psychosocial consequences due to their gambling behavior and meet criteria for pathological gambling behavior [7].

Following these premises, it is important that young people have realistic and factual knowledge of gambling since early adolescence. Indeed, it has been shown that gambling knowledge is significantly and negatively associated with gambling fallacies and problem gambling among adolescents [8]. Consequently, the importance of gambling knowledge for adolescents has been recognized in the prevention field. According to Messerlian et al. [9], the goal of universal prevention programs would be to increase knowledge and awareness of the risks and negative consequences of gambling for youth. In accordance with these positions, as reviewed by Ladouceur et al. [10], knowledge of gambling has been considered as one of the outcome variables to be modified both in gambling-specific prevention programs and in gambling and related skills programs. In detail, concerning the first type of interventions, programs have been developed by delivering factual information about gambling with the aim of improving adolescents' knowledge of gambling, and, consequently, arising in them a more negative attitude toward gambling [2,11-14]. Overall, these studies demonstrated that the presentation of a short video may increase knowledge about gambling in early and middle adolescence. Moreover, even in gambling and related skills programs, gambling knowledge has been taken into account, revealing a significant improvement in this dimension through the interventions $[8,15]$.

Despite the attention given to gambling knowledge in practice, relatively little attention has been focused on the way to assess this construct. The Questionnaire of Attitudes and Knowledge About Gambling, in its original French version, and English and Italian translations, has been the instrument used in most of the preventive interventions aimed at modifying adolescents' gambling knowledge [2,11-15]. The questionnaire contains one subscale measuring gambling knowledge, composed by sixteen Likert-type items with a 4-point scale ranging from totally disagree to totally agree. An example of item is "Lottery is a gambling activity". Despite its wide use, it is not an empirically evaluated instrument and, across the mentioned studies, the only psychometric information comes from Ferland, et al. [12], who reported that it has a two-factor structure with the first factor - knowledge about gambling - accounting for $18.6 \%$ of the variance,

${ }^{\star}$ Correspondence to: Maria Anna Donati, Department of Developmental and Social Psychology, Sapienza University of Rome, Rome, Italy, E-mail: marianna_donati@yahoo.it

Key words: gambling knowledge, adolescents, scale development, EFA, CFA, reliability, validity

Received: January 14, 2019; Accepted: January 30, 2019; Published: February 04,2019 
and the second factor, corresponding to the misconceptions about gambling, accounting for $12.9 \%$ of the variance. Additionally, they found an adequate Cronbach's alpha value (0.74) for the knowledge subscale, but an insufficient value for the misconceptions scale (0.58). Furthermore, Williams, et al. [8] assessed general knowledge of gambling and problem gambling with the 10-question Gambling Knowledge Scale, each scored one in case of correct answer, developed ad-hoc by the Authors, containing items referring to different aspects of gambling knowledge, as the legal age for purchase of lottery tickets and the potential addictive power of gambling. Although the Authors affirmed that the scale had good concurrent and predictive validity, no information was provided concerning its structural validity and reliability. In sum, there is a lack of a psychometrically sound instrument measuring adolescents' knowledge of gambling and problem gambling.

Given the important role of gambling knowledge, especially in the field of gambling prevention among youth, the aim of the present study was to develop a scale that accurately measures the awareness of some basic aspects of gambling among adolescents. This would offer benefits both for researchers and educators. Indeed, in gambling research, it would be important to assess gambling knowledge, and to include this aspect into theoretical models that are able to explain gambling behavior in young people. The final aim would be to derive precise and practical indications to conduct educational interventions [16]. From a practical point of view, in realizing preventive programs, identifying adolescents with poor knowledge about gambling can help with preventing future gambling-related cognitive distortions, as those adolescents can be treated with more extensive training activities focused on the meaning and the definition of gambling and its potential for addiction.

The instrument that we present in the current paper - the Gambling Related Knowledge Scale - For Adolescents (GRKS-A) - was developed to measure various aspects of gambling knowledge. In detail, our aim was to assess adolescents' awareness of the gambling definition, the nature of gambling outcomes, the functioning of gambling activities, and the riskiness of gambling activities for a potential addiction. Additionally, to provide a scale appropriate for large, multivariate studies in which many tests and scales need to be administered together, the GRKS-A was a short scale, in order to potentially offer added value [17]. Finally, we hypothesized to assess a unidimensional construct, viewed as a general knowledge of gambling. Thus, we expected to have a scale with a one-factor structure. One benefit of a unidimensional construct is that scoring is simplified as items are added for the whole domain to achieve the total score. A practical application of a unidimensional construct is designing a simple tool to measure gambling knowledge in the school setting that practitioners and operators could easily use to identify adolescents more lacking in gambling knowledge.

\section{Method}

\section{Construct Definition and Items Construction}

Development of the GRKS-A began with the preparation of detailed construct specifications describing various aspects of gambling. This work was conducted by analyzing the content items of existing scales to measure gambling knowledge, and by identifying different gambling aspects which are relevant to be known by adolescents in order to be aware of gambling characteristics. These aspects referred to the gambling definition, i.e., random activities with money to be bet, the nature of gambling outcomes, i.e., the unpredictability of results, losses more likely than winnings, the functioning of gambling activities, i.e., near misses and frequent small wins, and risks, and the dangerousness of gambling activities due to several structural characteristics and psychological mechanisms which reinforce gambling behavior. These specifications guided the creation of approximately 50 candidate items with a 4 -point Likert scale ( $1=$ totally disagree, $2=$ disagree, $3=$ agree, $4=$ totally agree). This item response format was selected because it was consistent with the need to avoid the potential tendency to select responses in the middle of a Likert scale and, at the same time, to assess if respondents have incorrect or correct knowledge about the different listed gambling aspects. Four experts (clinical psychologists with expertise in the field of adolescents gambling and one psychometrician) and six young adults and adolescents reviewed the items for content and grammar. As a result of this analysis, 10 items were modified and 18 items were removed because of ambiguous or repetitive stems. A 32 items scale was finally obtained in which items covered a broad range of contents deemed necessary to have an exhaustive knowledge of gambling.

\section{Participants}

Participants were 445 adolescents $(72 \%$ males, mean age $=16.81$, $\mathrm{SD}=0.84$, range $=15-20$ years) attending the $11^{\text {th }}$ grade of different high school in the North of Italy. Forty-seven percent of participants attended a technical school, $40 \%$ a lyceum, and $13 \%$ a vocational school. To obtain the approval of this research, a study protocol in accordance with the criteria of the Declaration of Helsinki was reviewed and approved by each Head Teacher and school board of different high schools of North Italy. Parents were informed with a short study description and asked to provide their informant consent. Written informed consent was obtained from all participants' parents, and their confidentiality was ensured.

\section{Measures}

The final version of the GRKS-A is a short self-report scale aimed at assessing adolescents' individual knowledge about gambling relative to its nature, functioning, and risks. It is composed of eight items with a 4 -point Likert scale (from $1=$ totally disagree, to $4=$ totally agree), to which participants can refer to indicate the extent to which they agree with what expressed in each statement. Total score consists of adding the values gained from each of the items. The higher the total score the higher gambling knowledge displayed. An example of item is "In gambling, small winnings stimulate people to gambling again".

The Gambling Related Cognitions Scale - Revised for Adolescents (GRCS-RA; Donati \& Primi, in preparation) is a self-report scale to assess gambling-related cognitions in young people $[18,19]$. In comparison with the original instrument, i.e., the GRCS, the revised form for adolescents contains fourteen Likert-type items having a 5-point scale ranging from 1 (strongly disagree) to 5 (strongly agree) [20]. Three specific gambling-related biases, according to Toneatto's model $[21,22]$, are measured by the following subscales: Illusion of Control (4 items), Predictive Control (6 items), and Interpretative Bias (4 items). In order to make the scale more appropriate and suitable for adolescents, items have been modified as to obtain thirdperson formulations (rather than first-person) that therefore could be administered to young people regardless their gambling behavior, contrary to the original scale, which was developed to be administered with adult gamblers. Examples of items are "Specific numbers and colors can help increase the chances of winning in gambling" (Illusion of Control), "In gambling, if you win once, you will surely win again" (Predictive Control), and "In gambling, people continue gambling despite losses because losses are due to bad luck and bad circumstances" (Interpretative Bias). The GRCS total score, obtained by summing the score for each item, was calculated. The total coefficient alpha for the current sample was satisfactory $(\alpha=0.87)$. 
The Gambling Attitude Scale [23,24] contains nine Likert-type items, using a 5-point scale ranging from strongly agree to strongly disagree. An example of an item is "You can make a living from gambling". Total scores on the scale were calculated so that high scores corresponded to an optimistic perception of gambling. The scale has been found to have good validity and reliability. Internal consistency for this scale was adequate in our sample $(\alpha=0.81)[23,24]$.

Section I of the Gambling Behavior Scale - For Adolescents consists of unscored items investigating gambling behavior, among which gambling frequency in the last 12 months [18]. Specifically, there are items assessing the frequency (never, sometimes in the year, sometimes in the month, sometimes in the week, daily) of participation during the last year in ten gambling activities (card games, bets on games of personal skill, bets on sports games, bets on horse races, bingo, slot machines, scratch cards, lotteries, online games, and private bets with friends). By summing responses to these ten items, a total score indicative of past-year gambling frequency can be obtained.

\section{Procedure}

Participants were recruited in schools. All participants completed their questionnaire through a self-administered procedure in class, during school-time; the time needed to complete it was approximately 35-40 minutes. The order of presentation was GRKS-A, GRCS-RA, GAS, and then the Section I of the GBS-A.

\section{Results}

\section{Item Selection}

The following process was used to reduce the original 32-item questionnaire to the final version. As a preliminary step, a missing analysis was conducted with the aim of deleting cases with more than $10 \%$ of missing values. No such case was found. For cases with less than $10 \%$ of missing values, missing was replaced with the mean value of the total sample. This replacement was conducted only for $2 \%$ of the participants $(\mathrm{n}=10)$. Then, all the items with loadings less than .40 were suppressed based on Stevens' suggestion that this cut-off point was appropriate for interpretative purposes [25]. This procedure led to delete 24 items. As subsequent step, the inter-item correlations for the remaining items were calculated. The aim of this was to examine, through item analysis, if there were some items were very similar to another [26]. Any redundant item was identified following this process. The final 8-item GRKS-A was thus obtained.

\section{Psychometric Properties of the final version of GRKS-A}

\section{Dimensionality}

To analyze the dimensionality of the final 8-item GRKS-A, univariate distributions of the items were examined for assessment of normality. Skewness and kurtosis indices were between -1 and +1 , except for in the case of item 1 , which was outside the range of normality (Table 1). However, deviation of one item from normality can be considered negligible [27].

An Exploratory Factor Analysis (EFA) with the Principal Axis Factoring (PAF) was performed on the 8-item scale. Kaiser's Meyer Olkin measure of sampling adequacy was 0.83 , showing that the patterns of correlation were relatively compact, and so factor analysis should produce distinct and reliable factors [28]. Bartlett's Test of Sphericity was significant $\left(\chi^{2}(28)=538.39, p<0.001\right)$, showing that there were some relationships between the variables. Oblique rotation was used because potential components were expected to be correlated.
Table 1. Means, standard deviations, skewness, and kurtosis of the eight items of the Gambling Related Knowledge Scale - For Adolescents (GRKS-A)

\begin{tabular}{|c|c|c|c|c|}
\hline Item & M & SD & Skewness & Kurtosis \\
\hline 1 & 3.57 & 0.71 & -1.88 & 3.51 \\
\hline 2 & 3.25 & 0.74 & -0.78 & 0.36 \\
\hline 3 & 3.43 & 0.76 & -1.35 & 1.52 \\
\hline 4 & 3.05 & 0.78 & -0.61 & 0.13 \\
\hline 5 & 3.31 & 0.66 & -0.91 & 1.54 \\
\hline 6 & 3.24 & 0.74 & -0.9 & 0.89 \\
\hline 7 & 3.28 & 0.73 & -0.85 & 0.56 \\
\hline 8 & 3.29 & 0.75 & -0.85 & 0.35 \\
\hline
\end{tabular}

Note. The Gambling Related Knowledge Scale - For Adolescents (GRKS-A) Likert scale is the following: 1 = "Totally Disagree", 2 = "Disagree", 3 = "Agree", 4 = "Totally Agree". $\mathrm{n}=445$

This analysis revealed two potential factors but all the items had factor loadings greater than 0.40 on one factor. The final factor analysis showed that a unidimensional solution was appropriate, with all 8 items having factor loadings greater than 0.40 , ranging from 0.44 to 0.60 , and explaining $25 \%$ of the variance.

Then, a Confirmative Factor Analysis (CFA) was carried out, employing the maximum likelihood (ML) method using AMOS [29]. Consistent with the results from the EFA, the one-factor model was tested. To verify the model's fit, the following indices were taken into account: The ratio of chi-square to its degrees of freedom $\left(\chi^{2} / d f\right)$; The comparative fit index; the Tucker-Lewis index; and the root mean square error of approximation [30-32]. In the case of $\chi^{2} / d f$, values below or equal to two are considered good, while values between two and three are considered acceptable [33]. For the TLI and CFI indices, values above .90 are indicative of acceptable fit, while values above 0.95 are indicative of excellent fit [34]. The RMSEA value is considered acceptable when it is below .08 and good when it is below 0.05 [35].

When we tested the fit of the one-factor model, the results showed a poor overall fit $\left(\chi^{2} / d f=4.43, \mathrm{TLI}=0.813, \mathrm{CFI}=0.867, \mathrm{RMSEA}=\right.$ $0.088,0.90 \%$ CI [0.070 - 0.107]). Modification indices (MIs) suggested adding error covariance between items 2 and 5, between items 1 and 3 , and between item 1 and 2 . Scrutiny of the content of items revealed a similarity in item content can lead to error covariances [36]. The modified model showed a better fit $\left(\chi^{2} / d f=2.35\right.$, TLI $=0.926$, CFI $=$ 0.955, RMSEA $=0.055, .90 \%$ CI $[0.033-0.078]$ ).

\section{Reliability and Validity}

Cronbach's alpha was 0.73 (95\% CI $[0.69-0.79])$ for the total scale. Following the cut-offs proposed by the European Federation of Psychologists' Association [37], the internal consistency value of the scale was adequate. All corrected item-total correlations were above 0.30 .

In order to analyze the criterion validity of the GRKS-A, we investigated its associations with gambling-related cognitive distortions, gambling economic perception, and gambling frequency. Results showed that the total score at the GRKS was significantly and negatively correlated with the total score at the GRCS $(r=-0.25, p$ $<0.001)$, with the total score at the GAS $(r=-0.47, p<0.001)$, and with the total score at the GBS-A Section I $(r=-0.25, p<0.001)$. The Pearson coefficient values indicated adequate or good validity following the cut-offs proposed by the EFPA [36]. Then, in order to provide proof of incremental validity of the GRKS-A, we examined its additional predictive power on gambling frequency with respect to the other variables, i.e., gambling-related cognitive distortions and gambling economic perception. To that aim, we conducted a stepwise linear regression analysis with the total score at the GBS-A Section 
Table 2. Incremental validity of the eight items of the Gambling Related Knowledge Scale - For Adolescents (GRKS-A) on gambling frequency

\begin{tabular}{|c|c|c|c|c|}
\hline Models & Predictors & $\boldsymbol{\beta}$ & $\boldsymbol{p}$ & $\mathbf{R}^{\mathbf{2}} \mathbf{A d j}$ \\
\hline Model 1 & GRCS total score & 0.2 & $<.001$ & 0.037 \\
\hline Model 2 & GRCS total score & 0.09 & 0.05 & 0.092 \\
\hline & GAS total score & 0.26 & $<.001$ & 0.057 \\
\hline & GRCS total score & 0.08 & 0.05 & \\
\hline & GAS total score & 0.21 & $<.001$ & \\
\hline & GRKS - A total score & -0.12 & 0.017 & \\
\hline
\end{tabular}

Note. GRCS $=$ Gambling-Related Cognitions Scale; GAS = Gambling Attitude Scale; GRKS-A = Gambling Related Knowledge Scale - For Adolescents $n=445$

I as dependent variable and the total scores at the GRCS, the GAS, and the GRKS-A as independent variables (Table 2). At the first step (Model 1), only the GRCS total score was entered in the regression analysis and it accounted for about $4 \%$ of the variance in gambling behavior, with gambling-related cognitive distortions as a significant and positive predictor. In Model 2, in addition to gambling-related cognitive distortions, the total score at the GAS was introduced, and there was a significant increase in explained variance $\left(\Delta R^{2}=0.057, p\right.$ $<0.001$ ), with gambling economic perception displaying a significant and positive predictive power on gambling frequency. In Model 3, the total score at the GRKS-A was added as predictor, and this led to a significant increase in explained variance $\left(\Delta R^{2}=0.012, p=0.017\right)$, with gambling knowledge having a significant and negative predictive power on gambling frequency, besides the significant and negative relationships between gambling-related cognitive distortions and gambling economic perception with gambling frequency. (Table 2)

\section{Discussion}

Several studies show that many adolescents gamble, and a considerable proportion of them develop pathological gambling behavior [3-5,7]. Among the reasons why adolescents gamble there is the fact that often youth are not aware of the technical definition of gambling activities, perceive gambling as a social and recreational activity, and they do not feel concerned about the potential negative consequences derivable from gambling [2,6]. Indeed, gambling knowledge has been found to be significantly and negatively associated with gambling fallacies and problem gambling among adolescents [8]. Additionally, improvement of adolescents' gambling knowledge has been one of the outcomes obtained through many preventive interventions $[2,8,11-15]$.

Nevertheless, a critical point concerns with the issue of measurement of adolescents' gambling knowledge, as the instruments used in the above cited interventions have not been well investigated from a psychometric point of view. As a result, nowadays there is a lack of measurement tools with adequate psychometric properties to assess gambling knowledge in adolescents. To fulfill this gap, the aim of the present study was to develop a new instrument able to evaluate this specific construct with a sample of Italian adolescents. In detail, our aim was to create a short and unidimensional scale in order to offer potential benefits for researchers and practitioners. After the developmental process, the new-developed scale - the GRKS-A resulted to be a unidimensional, and reliability index and validity analysis were satisfactory. In particular, we showed that gambling knowledge measured through the GRKS-A had a significant and negative predictive power on gambling frequency, besides the significant and negative relationships between gambling-related cognitive distortions and gambling economic perception with gambling frequency.

In terms of practical implications, the GRKS-A's observed psychometric properties allow for its use to measure gambling- related knowledge in research and practice involving adolescents. The instrument can therefore help practitioners in the early identification of at-risk adolescents characterized by low levels of knowledge and awareness of gambling risks, and in planning educational interventions. Indeed, it may be used as a pre- and post-intervention measure in order to verify if significant improvement in knowledge occur because of the intervention. Its brevity and unidimensionality offer advantages for this use. Moreover, as it would be important to know detailed information about gambling activities from an early age, the scale may be used also with children, among which some studies have tried to investigate gambling behavior and perceptions [38-40].

This study offers several notable strengths, including the large sample size, and the development and psychometric analysis of a new scale to measure gambling knowledge in adolescents. However, there are also some limitations. First, the findings of this study were based on self-report data, so it does not necessarily follow that participants' responses completely corresponded to their actual gambling behavior. Second, this was a cross-sectional study involving a sample of publicschool students, thus, generalizability to other populations is limited. Future research should focus on students from different age groups (e.g., middle school or primary students) or on adolescents who do not attend high school. Finding equivalent psychometric properties with these specific samples would allow for generalization of the use of the scale with a wide range of youth. Finally, as this study has been conducted with Italian adolescents, and some limitations regarding external validity might be related to the specificity of the sample. For this reason, it should be desirable to test the psychometric properties of the scale in samples of adolescents belonging to different cultures and languages.

Despite the limitations, overall our results provide evidence that the Italian version of the GRKS-A for juveniles appears psychometrically appropriate to be used by researchers and practitioners dealing with the issue of research and prevention of problem gambling among adolescents.

\section{Acknowledgments}

The project "Azzardo BUGIArdo" was supported by Regione Lombardia (n. 2379).

\section{References}

1. Ladouceur R, Ferland F (2003) Prevention of problem gambling among youths. In Gullotta TP, Bloom M (Eds) The Encyclopedia of primary prevention and health promotion, Kluwer, Newyork.

2. Ladouceur R, Ferland F, Vitaro F (2004) Prevention of problem gambling: Modifying misconceptions and increasing knowledge among youths. Journal of Primary Prevention 25: 329-336.

3. Donati MA, Chiesi F, Primi C (2013) A model to explain at-risk/problem gambling among male and female adolescents: Gender similarities and differences. $J$ Adolesc 36: 129-137. [Crossref]

4. Turner NE, Ialomiteanu A, Paglia-Boak A, Adlaf EM (2011) A typological study of gambling and substance use among adolescent students. Journal of Gambling Issues 25: 88-107. 
5. Splevins K, Mireskandari S, Clayton K, Blaszczynski A (2010) Prevalence of adolescent problem gambling, related harms and help-seeking behaviours among an Australian population. J Gambl Stud 26: 189-204. [Crossref]

6. Ladouceur R, Ferland F, Vitaro F, Pelletier O (2005) Modifying youths' perception toward pathological gamblers. Addictive Behaviors 30: 351-354.

7. Calado F, Alexandre J, Griffiths MD (2017) Prevalence of Adolescent Problem Gambling: A Systematic Review of Recent Research. J Gambl Stud 33: 397-424. [Crossref]

8. Williams RJ, Wood RT, Currie SR (2010) Stacked Deck: An Effective, School-Based Program for the Prevention of Problem Gambling. J Prim Prev 31: 109-125. [Crossref]

9. Messerlian C, Derevensky JL, Gupta R (2005) Youth gambling problems: a public health perspective. Health Promot Int 20: 69-79. [Crossref]

10. Ladouceur R, Goulet A, Vitaro F (2013) Prevention programmes for youth gambling: a review of the empirical evidence. International Gambling Studies 13: 141-159.

11. Capitanucci D, Smaniotto R, Biganzoli A (2010) La prevenzione del gioco d'azzardo problematico negli adolescenti attraverso l'adattamento del video Lucky. Quaderno Italiani di Psichiatria 29: 30-39.

12. Ferland F, Ladouceur R, Vitaro F (2002) Prevention of problem gambling: modifying misconceptions and increasing knowledge. J Gambl Stud 18: 19-29. [Crossref]

13. Gaboury A, Ladouceur R (1993) Evaluation of a prevention program for pathologica gambling among adolescents. J Prim Prev 14: 21-28. [Crossref]

14. Lavoie MP, Ladouceur R (2004) Prevention of gambling among youth: Increasing knowledge and modifying attitudes toward gambling. eGambling: The Electronic Journal of Gambling Issues 1-10.

15. Donati MA, Primi C, Chiesi F (2014) Prevention of problematic gambling behavior among adolescents: Testing the efficacy of an integrative intervention. J Gambl Stud 30: 803-818. [Crossref]

16. Keen B, Blaszczynski A, Anjoul F (2017) Systematic Review of Empirically Evaluated School-Based Gambling Education Programs. J Gambl Stud 33: 301-325. [Crossref]

17. Ziegler M, Kemper CJ, Kruyen P (2014) Short scales-Five misunderstandings and ways to overcome them. Journal of Individual Differences 35: 185-189.

18. Donati MA, Primi C (2017) In preparation. A new scale to measure gambling-related cognitive distortions in adolescents.

19. Donati MA, Ancona F, Chiesi F, Primi C (2015) Psychometric properties of the Gambling Related Cognitions Scale (GRCS) in young Italian gamblers. Addict Behav 45: 1-7. [Crossref]

20. Raylu N, Oei TP (2004) The Gambling Related Cognitions Scale (GRCS): Development, confirmatory factor validation and psychometric properties. Addiction 99: 757-769. [Crossref]

21. Toneatto T (1999) Cognitive psychopathology of problem gambling. Subst Use Misuse 34: 1593-1604. [crossref]

22. Toneatto T, Blitz-Miller T, Calderwood K, Dragonetti R, Tsanos A (1997) Cognitive distortions in heavy gambling. Journal of Gambling Studies 13: 253-266. [Crossref]
23. Delfabbro P, Thrupp L (2003) The social determinants of youth gambling in South Australian adolescents. J Adolesc 26: 313-330. [Crossref]

24. Primi C, Donati MA, Bellini I, Busdraghi C, Chiesi F (2013) Measuring the attitude towards the profitability of gambling: The psychometric properties of the Gambling Attitude Scale. BPA-Applied Psychology Bulletin (Bollettino di Psicologia Applicata) 266: 49-58.

25. Stevens JP (1992) Applied Multivariate Statistics for the Social Sciences. (2nd Edn) Erlbaum, Hillsdale, NJ.

26. DeVellis RF (1991) Applied social research methods series. Scale development: Theory and applications. Thousand Oaks, CA, US.

27. Ghasemi A, Zahediasl S (2012) Normality tests for statistical analysis: a guide for non-statisticians. International Journal of Endocrinology and Metabolism 10: 486-489. [Crossref]

28. Field A (2009) Discopering Statistics Using SPSS. (3 Edn).

29. Arbuckle J, Wothke W (1999) AMOS 4 user's reference guide. Smallwaters Corporation, Chicago.

30. Bentler PM (1990) Comparative fit indexes in structural models. Psychol Bull 107: 238-246. [Crossref]

31. Tucker LR, Lewis C (1973) A reliability coefficient for maximum likelihood factor analysis. Psychometrika 38: 1-10.

32. Steiger JH, Lind JC (1980) Statistically based tests for the number of common factors In annual meeting of the Psychometric Society, Iowa City, IA 758: 424-453.

33. Schermelleh-Engel K, Moosbrugger H, Müller H (2003) Evaluating the fit of structural equation models: Tests of significance and descriptive goodness-of-fit measures. Methods of Psychological Research Online 8: 23-74.

34. Hu LT, Bentler PM (1999) Cutoff criteria for fit indexes in covariance structure analysis: Conventional criteria versus new alternatives. Structural Equation Modeling: A Multidisciplinary Journal 6: 1-55.

35. Kline RB (2010) Principles and Practice of Structural Equation Modeling (3rd Edn.) New York: Guilford Press.

36. Byrne BM (2004) Testing for multigroup invariance using AMOS graphics: A road less traveled. Structural Equation Modeling 11: 272-300.

37. Evers A, Muñiz J, Hagemeister C, Høtmælingen A, Lindley P, et al. (2013) Assessing the quality of tests: revision of the EFPA review model. Psicothema 25: 283-291. [Crossref]

38. Pagani LS, Derevensky JL, Japel C (2009) Predicting gambling behavior in sixth grade from kindergarten impulsivity. Arch Pediatr Adolesc Med 163: 238-243. [Crossref]

39. Pitt H, Thomas SL, Bestman A, Daube M, Derevensky J (2017) Factors that influence children's gambling attitudes and consumption intentions: lessons for gambling harm prevention research, policies and advocacy strategies. Harm Reduct J 14: 11. [Crossref]

40. Temcheff CE, Déry M, St-Pierre RA, Laventure M, Lemelin JP (2016) Precocious initiation into smoking, alcohol use, and gambling among children with conduct problems. Can J Psychiatry 61: 50-58. [Crossref]

Copyright: (C2019 Donati MA. This is an open-access article distributed under the terms of the Creative Commons Attribution License, which permits unrestricted use, distribution, and reproduction in any medium, provided the original author and source are credited. 